\title{
Pathogenesis of Multiple Squamous Cell Carcinomas in the Oral Cavity: Clinicopathological and immunohistochemical study of 31 patients
}

\author{
Yenchun Liu ${ }^{1}$, Tran Tu Ngoc ${ }^{1}$, Norihiko Okada ${ }^{2}$ and Minoru Takagi ${ }^{1}$ \\ ${ }^{1}$ Molecular Pathology, ${ }^{2}$ Diagnostic Oral Pathology, Oral Restitution, Oral Health Sciences, Graduate School, Tokyo Medical and \\ Dental University, Tokyo, Japan
}

Liu Y, Ngoc T, Okada N and Takagi M. Pathogenesis of multiple squamous cell carcinomas in oral cavity: Clinicopathological and immunohistochemical study of 31 patients. Oral Med Pathol 2003; 8: 75-82, ISSN 1342-0984

\begin{abstract}
Carcinomas of the oral cavity sometimes arise as multiple, independent lesions and this observation has led to the concept of field cancerization. Howerever, the mechanism of multiple occurrences of oral squamous cell carcinoma (OSCC) is still unknown. Thirty-one cases of synchronous and/or metachronous multiple OSCCs were selected from biopsy and operation materials of the Dental Hospital, Tokyo Medical and Dental University. Histopathogenetic study and investigation were carried out. The clonality of the tumor tissues was analyzed by immunohistochemistry for Ki-67, Bcl-2, p53, cyclinB1 and cyclinD1. Histologic observation showed two patterns of occurrence of OSCC: de novo type and premalignancy-associated type. Twenty-one patients with multiple OSCCs were classified into 3 groups. Group I was de novo type, Group II was mixed type and Group III was premalignacy-associated type. Seventeen of 21 patients $(80.9 \%)$ revealed p53-positivity in the epithelium apart from the tumor. Fifty-two of 69 tumors (75.4\%) showed a different expression rate between Cyclin B1 and Cyclin D1. This data suggests that those patients are at high risk of developing second primary tumors. It also suggests the possibility of polyclonal development of the multiple OSCCs.
\end{abstract}

Key words: multiple carcinomas, field cancerization, oral cavity, squamous cell carcinoma, immunohistochemistry

Correspondence: Minoru Takagi, Molecular Pathology, Oral Restitution, Oral Health Science, Graduate School, Tokyo Medical and Dental University, 1-5-45 Yushima, Bunkyo-ku, Tokyo 1138549, Japan

Phone: +81-3-5803-5451, Fax: +81-3-5803-0188, E-mail: m-takagi.mpa@tmd.ac.jp

\section{Introduction}

Patients with squamous cell carcinoma of the oral cavity sometimes show development of multiple independent carcinomas synchronously or metachronously. This finding led to the concept of field cancerization as presented by Slaughter et al. in 1953 (1), in which an area of epithelium has been widely preconditioned by long-term exposure to carcinogens. However, the pathogenesis and molecular genesis of multiple occurrence OSCCs are still unknown. The development of multiple carcinomas is hypothesized to be related either to independently-occurring genetic events in separate regions or to the malignant transformation of single cells that give rise to genetically-related carcinomas in distinctly separate portions, also known as intramucosal migration. The former is of polyclonal origin (also called a second primary tumor) and the latter is of monoclonal origin (a second field tumor)(2). Recent investigations of field cancerization have been carried out using loss of heterozygosity ( $\mathrm{LOH})$ pattern analysis, fluorescence in situ hybridization (FISH) analysis, microsatellite analysis and $\mathrm{X}$ chromosome inactivation analysis (3-11)

In the present study, we analyzed histologically the pathogenesis of multiple occurrence OSCCs and used the immunohistochemical expression rates of Ki-67, Bcl-2, p53, cyclinB1 and cyclinD1 as biomarkers in order to clarify clonality of the multiple OSCCs.

\section{Material and methods \\ Case materials}

Thirty-one patients with multiple OSCCs provided the materials used in the present study. The materials were collected from the biopsy and operation material files of the Department of Diagnostic Oral Pathology and 
Molecular Pathology, Tokyo Medical and Dental University, from 1991 to 2000 . The definition of multiple OSCCs is based on the criteria of Warren and Gates (12); a new carcinoma with different histologic type that occurred more than 3 years after treatment; another tumor separated from the initial primary tumor at a distance of more than $2 \mathrm{~cm}$ of clinically normal epithelium. Localization of SCCs and premalignant lesions of the oral cavity in the present 31 cases are shown in Table 1 . Regarding the location of primary carcinoma, 11 cases were located in the buccal mucosa, 9 cases in the lower gingiva, 5 cases in the upper gingiva and tongue each, and one case in the palate and lips, respectively. About the location of second primary tumor, there were 9 cases in the lower gingiva, 7 cases in the buccal mucosa, 5 cases in the upper gingiva and tongue, each, two cases in the palate and floor of the

Table 1: Localization of SCCs and premalignant lesions of the oral cavity

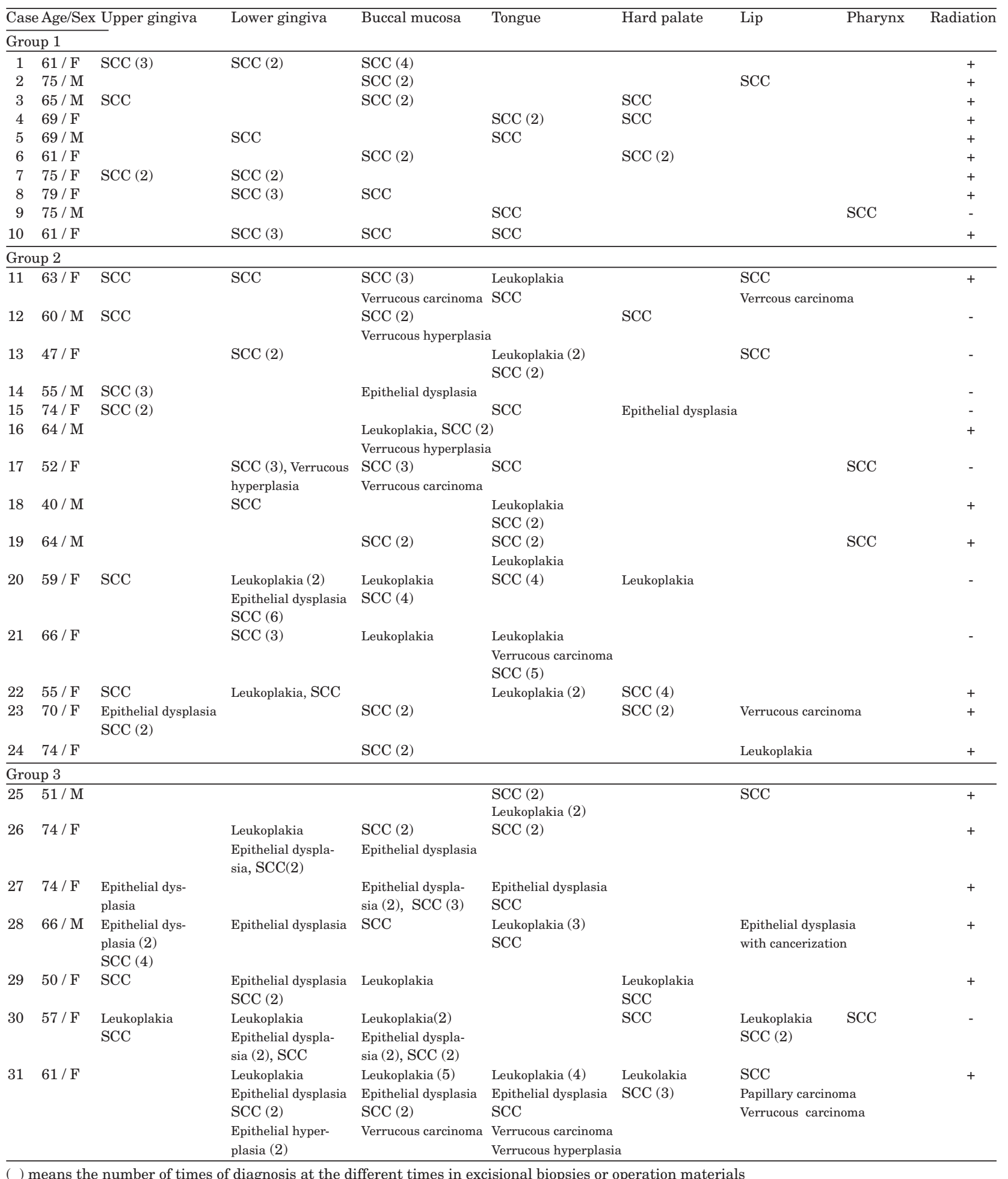

( ) means the number of times of diagnosis at the different times in excisional biopsies or operation materials 
mouth, respectively, and one case in the lip and pharynx, each. Combinations of primary and secondary tumor of multiple tumors are shown in Table 2 . Those 31 patients were classified into 3 groups according to the criteria mentioned later. The first group (Group I) consisted of 10 patients $(32.3 \%)$. The second group (Group II) consisted of 14 patients (45.2\%) and the third group (Group III) consisted of 7 patients (22.6\%), respectively. The average age was 69.0 years in Group I, 60.2 years in Group II and 61.9 years in Group III. The male to female ratio was 4:6 in Group I, 5:9 in Group II, and 2:5 in Group III. Radiotherapy was performed in 9 cases of Group I, in 7 cases of Group II and in 6 cases of Group III. Most of the patients in all groups were non-smokers. Histological examination was performed by using the specimens stained by hematoxylin and eosin.

\section{Immnunohistochemistry}

Two distinctly separate OSCCs of 21 patients were selected to compare with the results of immunohistochemical stainability of Ki-67, Bcl-2, p53, Cyclin B1 and Cyclin D1.

Paraffin-embedded, $4 \mathrm{~m}$-thick tissue sections were studied immunohistochemically, using a primary mouse monoclonal antibody (DAKO) for Ki-67, Bcl-2, p53, cyclinB1 and cyclinD1 by SAB (Streptoavidin-biotinylated peroxidase complex) methods. Slides were dried at $37^{\circ} \mathrm{C}$ for $18 \mathrm{hr}$. and then deparaffinized through a series of xylene baths. The samples were rehydrated in graded alcohol series. To retrieve the antigenicity, the tissue sections for Bcl-2 staining were treated with microwave in $10 \mathrm{mM}$ citrate buffer (pH6.3), three times for 5 min. each, and the sections for Ki-67, p53, CyclinB1 and CyclinD1 were heated by autoclave $\left(120^{\circ} \mathrm{C}\right)$ in $10 \mathrm{mM}$ citrate buffer (pH6.3) for $15 \mathrm{~min}$. After cooling for $30 \mathrm{~min}$. at room temperature, the sections were then immersed in methanol containing $0.3 \%$ hydrogen peroxidase for $20 \mathrm{~min}$. to block the endogenous perxidase activity and were incubated in $2.5 \%$ normal rabbit-serum to reduce nonspecific immunoreactions. Sections were incubated with a monoclonal antibody Bcl-2 (DAKO) at a 1:50 dilution; Ki-67, p53, CyclinB1 and CyclinD1 at 1:100 dilution (Table 3). All incubations were carried out overnight in moist chambers at $4^{\circ} \mathrm{C}$. Sections were processed using standard SAB staining method, according to the manufacturer's recommendations (DAKO). 3,3'-diamino benzidine (DAB) was used as a chromogen, and commercial hematoxylin was used for counterstaining. As a negative control, normal mouse serum and PBS were used instead of the first antibody. Staining results were classified into 4 grades: no reactivity (-); 1-10\% tumor cells positive (+); 10-50\% tumor cells positive $(++)$; and $50-100 \%$ tumor cells positive $(+++)$.

\section{Results}

\section{Histopathological findings of multiple OSCCs}

Thirty-one patients with multiple OSCCs in the present study were classified into three groups according to clinical features and microscopic findings. Group I was a de novo type, and in this group carcinoma developed without specific changes in the adjacent normal epithelium (Fig 1a). Sometimes adherent phenomenon between carcinoma cells and normal covering epithelium was seen, but this was secondary to the carcinoma. Group II was a mixed type, and cancerization occurred with de novo and in association with premalignant lesions. The typical case was pictured in Fig. 2, case 20. Group III was a premalignancy-associated type. As shown in (Figs. 1b, 1c, 1d) there could be seen mild, moderate and severe dysplasia and carcinoma in situ. In the operation materials of OSCCs, we could sometimes find multiple early carcinoma associated with premalignant lesions (Fig 1d). The typical case was pictured in Fig. 2, case 31.

Table 3: Monoclonal antibodies used

\begin{tabular}{cccc}
\hline Antibody & $\begin{array}{c}\text { Code No. } \\
\text { (DAKO) }\end{array}$ & $\begin{array}{c}\text { Antigen } \\
\text { retrieval }\end{array}$ & Dilution \\
\hline $\begin{array}{c}\text { Monoclonal mouse } \\
\text { Anti-Ki67 }\end{array}$ & M 7187 & Auto. & $1: 100$ \\
$\begin{array}{c}\text { Monoclonal mouse } \\
\text { Anti-Bcl2 }\end{array}$ & M 0887 & Micro. & $1: 50$ \\
$\begin{array}{c}\text { Monoclonal mouse } \\
\text { Anti-p53 Oncoprotein } \\
\text { Monoclonal mouse } \\
\text { Anti-Cyclin B1 }\end{array}$ & M 7001 & Auto. & $1: 100$ \\
$\begin{array}{c}\text { Monoclonal mouse } \\
\text { Anti-Cyclin D1 }\end{array}$ & M 3530 & Auto. & $1: 100$ \\
\hline
\end{tabular}

Auto: autoclave, Micro: microwave
Table 2: Combination of primary and secondary tumors

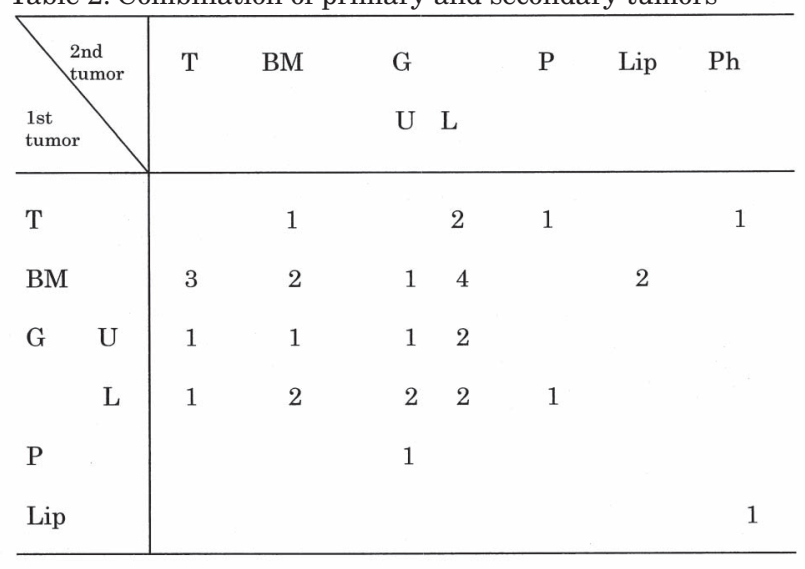

T: tongue, BM: buccal mucosa, G: gingiva; U: upper, L: lower, P: palate Ph: pharynx 
Immunohistochemical expression rate of multiple OSCCs We analyzed 21 (69 tumors) of 31 patients whose materials could be sectioned from the paraffin-embeded blocks. In Group I, there were 6 patients (21 tumors), in Group II 9 patients (31 tumors) and in Group III 6 patients (17 tumors).

Sixty-three of 69 tumors (91.3\%) revealed Ki-67 positivity. Bcl-2 immunoreactivity was found in 36 of 69 tumors (52.2\%). There was only one tumor which revealed $(+++)$, while 7 tumors $(10.1 \%)$ were $(++), 28$ tumors $(46 \%)$ were $(+)$ and 33 tumors (47.7\%) showed no reaction (Table 4). Seventeen of 21 patients ( $80.9 \%$ ) showed significant differences of the expression rate of p53 in the multiple anatomical sites within the same patient. Relationship between p53 and Bcl-2 expression was found in 30/69 tumors $(43.5 \%)$ for p53+/Bcl2+, in 27/69 tumors (37.7\%) for p53+/Bcl-2-, in 6/69 tumors (8.7\%) for p53-/ Bcl-2+, and in 6/69 tumors (8.7\%) for p53-/Bcl-2-. Thirty of 57 p53(+) tumors (52.6\%) revealed Bcl-2(+) (Table 4). Sixtythree of 69 tumors $(91.3 \%)$ revealed Cyclin B1(+), 51 of 69 tumors (82.6\%) revealed Cyclin D1(+) and 57 of 69 tumors $(82.6 \%)$ revealed p53(+). There were 17 of 69 tumors $(24.6 \%)$ that showed the same expression rate with Cyclin B1 and Cyclin D1 (e.g. Cyclin B1(+++ ) / Cyclin D1(+++), Cyclin B1 (++ ) / Cyclin D1 (++ ) or Cyclin B1 (+ ) / Cyclin D1 (+) ). Fifty-two of 69 tumors (75.4\%) showed a different expression rate between Cyclin B1 and Cyclin D1. Significant differences in expression rate were observed between Cyclin B1 and Cyclin D1. There were 25 of 69 tumors $(36.2 \%)$ that showed the same expression rate between Ki-67 and Cyclin B1 (e.g. Ki-67 (+++) / Cyclin B1(+++), Ki-67 (++) / Cyclin B1 (++), Ki-67 (+) / Cyclin B1 (+) or Ki-67(-) / Cyclin B1(-) ). Forty-four of 69 tumors $(63.8 \%)$ showed a different expression rate between $\mathrm{Ki}$ 67 and Cyclin D1. The relationship of the immunohistochemical expression rate between two distinct separate OSCCs in one patient are given in Table 5. A different expression rate was seen in 13/21 (61.9\%) in Ki-67, in 10/ $21(47.6 \%)$ in Bcl-2, in 12/21 (57.1\%) in p53, in 15/21 (71.4\%) in Cyclin B1 and in 14/21 (66.7\%) in Cyclin D1 (Table 6). In Group I, Ki-67 was different in 5/6, Bcl-2 in $3 / 6$, p53 in 3/6, Cyclin B1 in 3/6 and Cyclin D1 in 3/6. In Group II, Ki-67 was different in 5/9, Bcl-2 in 3/9, p53 in 5/ 9, Cyclin B1 in 8/9 and Cyclin D1 in 6/9. In Group III, Ki67 was different at 3/6, Bcl-2 in 4/6, p53 at 4/6, Cyclin B1 in 4/6 and Cyclin D1 in 5/6 (Table 7). In conclusion, differences in expression rate were also significant between Cyclin B1 and Cyclin D1.

\section{Discussion}

In the present study, we studied the pathogenesis of multiple occurrences of squamous cell carcinoma in the oral cavity. Slaughter et al. (1) proposed the concept of field cancerization by citing the following observations: 1) several distinct lesions within the same specimen, 2) margins of normal mucosa around a tumor with another noncontiguous area of dysplasia, 3) cellular heterogeneity of the tumor, 4) a larger superficial extension than depth, and 5) the high incidence of second primary tumors found in one patient. However, the field cancerization theory cannot explain pathogenesis of all OSCCs. Our observations suggested two patterns of histogenesis i.e. de novo carcinogenesis and premalignancy-associated carcinogenesis. Several distinct lesions within the same specimen could be found in the carcinogenesis of the premalignant lesions, as shown in Fig. 1d. The margin of the normal mucosa around the carcinoma was basically normal without dysplasia in de novo carcinogenesis, as shown in Fig. (1a). A superficial spreading carcinoma, which shows a larger superficial extension than depth, is one growth pattern of OSCC, though it is not frequent. Our observation of about 1,350 cases of OSCCs, which were newly registered in our laboratory from 1991 to 2000 , suggested that de novo cancerization might be most common in OSCCs. The incidence of multiple OSCCs was also low and probably less than $10 \%$ (2).

Comparative molecular analysis of premalignant and malignant lesions is very important for determining the nature and the stage of genomic alterations in the process of cancerization. Recently, mechanisms of cancerization and field cancerization have been studied mainly by pattern analysis of loss of heterozygosity $(\mathrm{LOH})(4,5$, $7,10-11$ ). Considering pathogenesis of multiple OSCCs, the most basic problem is whether carcinogenesis is monoclonal or polyclonal in origin $(2,3,6,13-16)$. As markers for determining clonality of the distinct separate carcinomas, pattern of $\mathrm{LOH}$ and mutation sites of p53 were mainly used (4-5, 7-8, 11, 17-25). If there could be the same pattern of $\mathrm{LOH}$ or the same site of p53 mutation in the distinct separate carcinoma, it has been stated that these two separate carcinomas might be of monoclonal origin $(3,7,10,13-15,17-18)$. However, this conclusion might be not yet valid because there are variations in the patterns of $\mathrm{LOH}$ and sites of p53 mutation.

In the present study, we used immunohistochemical analysis of anti-Ki67, Bcl-2, p53, cyclinB1 and cyclinD1 positivity in the tumor cells in order to analyze the clonality of carcinomas. Homann et al. (25) have shown that in multiple anatomical sites within one cancer patient, histologically normal appearing epithelial cells apart from the tumor can be found that express mutated p53 protein. This finding, which was confirmed by Waridel et al. (17) has provided a possible molecular basis for the development of second (multiple) primary tumors. Overexpression of p53 in the epithelium apart from the tumor was associated with an increased incidence of second primary carcinoma. At the same time, this provides strong evidence indicating a correlation between the p53 protein status in the primary tumor and the occurrence of second primary tumors (25). In this study, $80.9 \%$ of all 


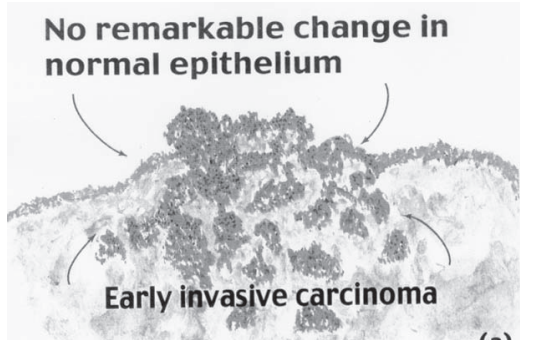

(a)

(a) Early de novo carcinogenesis. The epithelium adjacent to the original carcinoma shows no remarkable change. Group I.

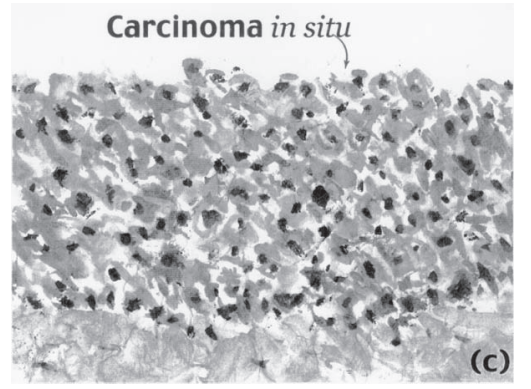

(c) Carcinoma in situ appears in premalignancy-associated carcinogenesis. Usually the degree of epithelial dysplasia increases in the time course. This shows the background of field cancerization. Group II.

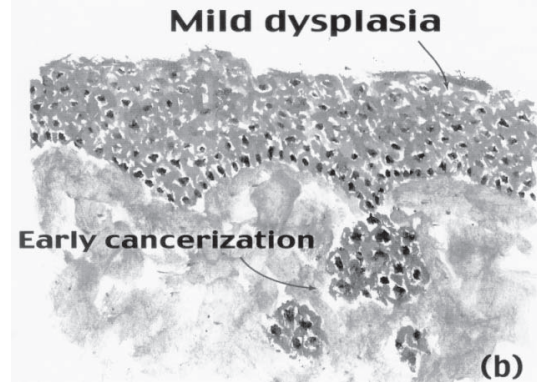

(b) Early cancerization sometimes occurs even from mild epithelial dysplasia of the oral mucosa in premalignancyassociated carcinogenesis.

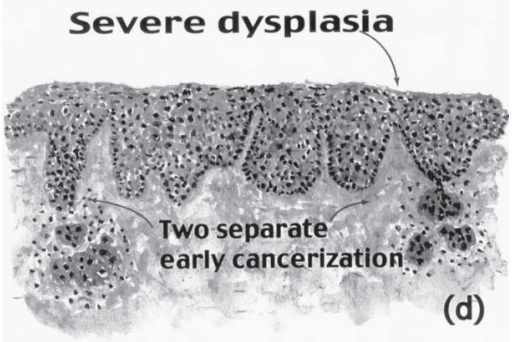

(d) Early cancerization sometimes occurs as multiple carcinomas in the separate regions. This explains the mode of multiple occurrences. In Group III, histologic features of b, $c$ and d are seen.

Fig. 1: Scheme of histogenesis of OSCCs

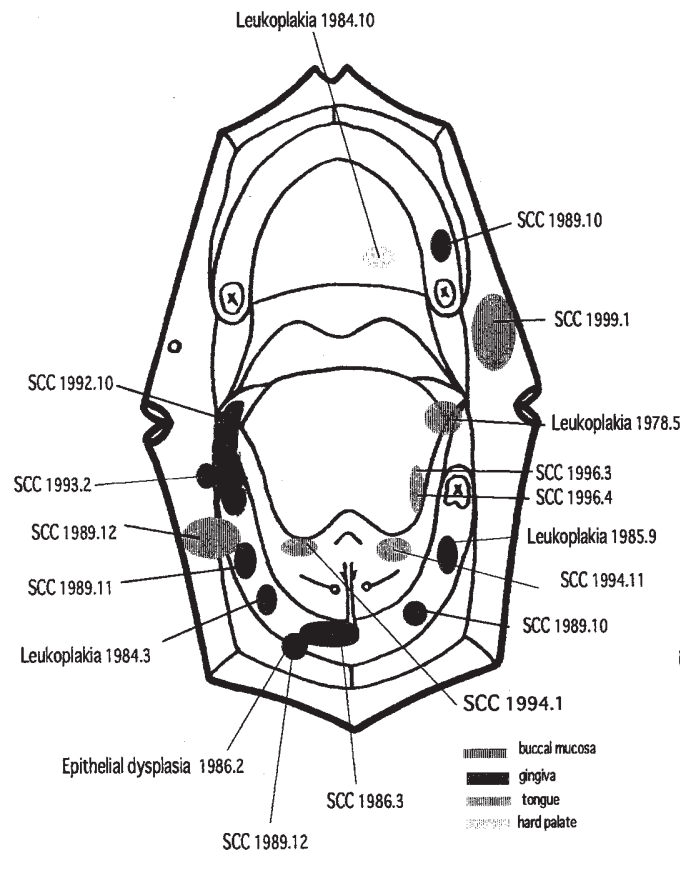

CASE 20

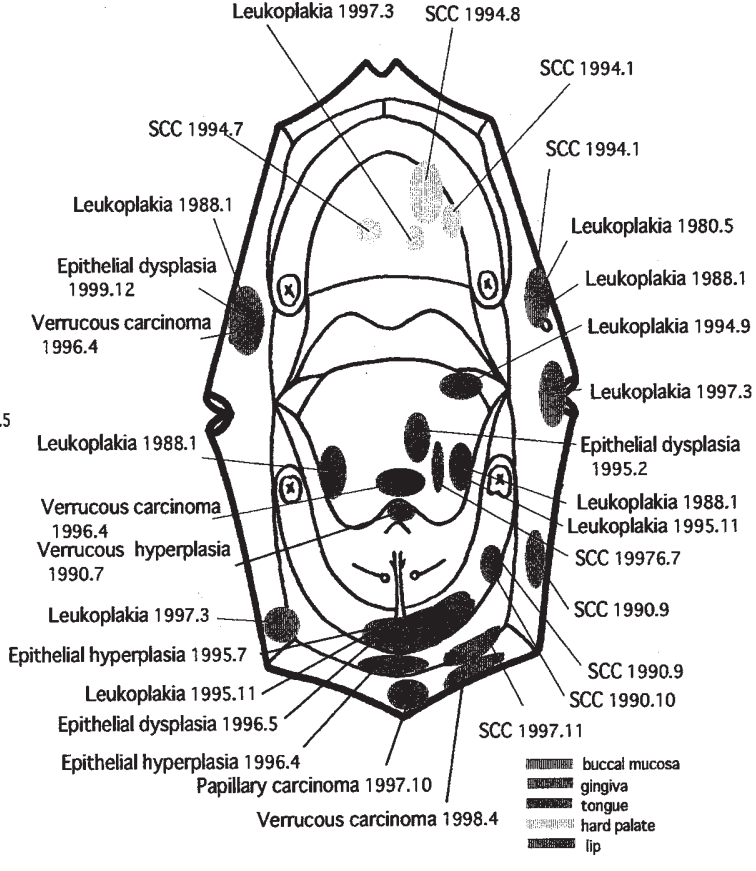

CASE 31

Fig. 2: Distribution of SCCs and premalignant lesions.

Numbers indicate date of pathological diagnosis of the lesions

Case 20: a 59-year-old woman visited our hospital in May 1978. (1) In 1978, white patch appeared on the floor of the mouth, and the pathological diagnosis was leukoplakia. (2) In 1984, leukoplakia appeared on the right mandibular gingiva and palate. (3) In 1986, SCC appeared on the right lower gingiva. (4) In 1989, other SCCs developed on the left upper gingiva and left lower gingiva. (5) In 1992, papillary SCC appeared on the lower gingiva. (6) In 1994, another SCC appeared on the right floor of mouth. (7) In 1996, SCCs were found on the left side of the tongue and on the left buccal mucosa.

Case 31: a 54-year-old woman visited our hospital in May 1980. (1) In 1980, leukoplakia appeared on the left buccal mucosa. (2) In 1988, four separate leukoplakias appeared on the left lateral surface of tongue, right side of tongue, left buccal mucosa and right buccal mucosa. (3) In 1990, papillary SCC appeared on the left lower gingiva and (4) In 1994, two separate SCCs appeared on the left palate and left buccal mucosa. (5) In 1995, severe dysplasia with early cancerization was found on the left lateral surface of tongue. (6) In 1997, SCCs were appeared on the vestibulum oris and right buccal mucosa. 
cases expressed detectable levels of p53 in epithelium apart from the tumor. This suggests that the second tumors occurred in these patients, which were new tumors not be caused by metastasis of the primary tumor. Overexpression of the p53 protein is frequently, but not always, associated with gene mutation and is regarded a

Table 4: Expression rate of Bcl-2 and relationship between p53 and Bcl-2 expression

\begin{tabular}{|c|c|c|}
\hline Expression rate of Bcl-2 & No.of OSCCs & $\%$ \\
\hline Bcl-2+++ & 1 & 1.4 \\
\hline Bcl-2++ & 7 & 10.1 \\
\hline Bcl-2+ & 28 & 46.0 \\
\hline Bcl-2- & 33 & 47.7 \\
\hline $\begin{array}{l}\text { Relationship between p53 } \\
\text { and Bcl-2 expression }\end{array}$ & No. of OSCCs & $\%$ \\
\hline p53+ / Bcl-2+ & 30 & 43.5 \\
\hline p53+ / Bcl-2- & 27 & 37.7 \\
\hline p53- / Bcl-2+ & 6 & 8.7 \\
\hline p53- / Bcl-2- & 6 & 8.7 \\
\hline
\end{tabular}

OSCCs: oral squamous cell carcinomas promising candidate that might predict patient prognosis(26). p53 overexpression not only occurs in $23-30 \%$ of all head and neck tumors, but also has commonly been found in dysplastic and nondysplastic premalignant epithelial lesions(26). Bcl-2 expression was often observed in the same area of the tumor. Xin Xie et al. (27) revealed that high expression of Bcl-2 was significantly associated with poor prognosis and low expression with good prognosis. p53 has been suggested to induce apoptosis through regulation of Bcl-2 gene expression. In this study, most cases showed low expression of Bcl-2, and 30 of the p53(+) 57 tumors $(52.6 \%)$ revealed Bcl-2(+), which indicates that tumors were with good prognosis. Cyclin B1 plays an important role in control of $\mathrm{G}_{2}-\mathrm{M}$ transition of the cell cycle, and Cyclin B1 has a direct effect on mitosis. Overexpression of CyclinB1 may lead to uncontrolled cell proliferation, which is a characteristic of SCC of the oral cavity $(28,29)$. Poor correlation between Cyclin B1 and $\mathrm{Ki}-67$ staining is likely to be an indication of aberrant cell cycle progression at G2/M transition in oral carcinoma(30). In this study we also obtained the following results: 63 of 69 tumors (91.3\%) were CyclinB1 positive. A different expression rate of Cyclin $\mathrm{B} 1$ between two distinctly separate sites in the same patient was shown in Fig. 3. Cyclin D1 is an unstable protein essential for G1 progression(31). Cyclin D1 expression is seen late in the development of oral premalignant and malignant lesions.

Table 5: Relationship of immunohistochemical findings between two distinctly separate OSCCs

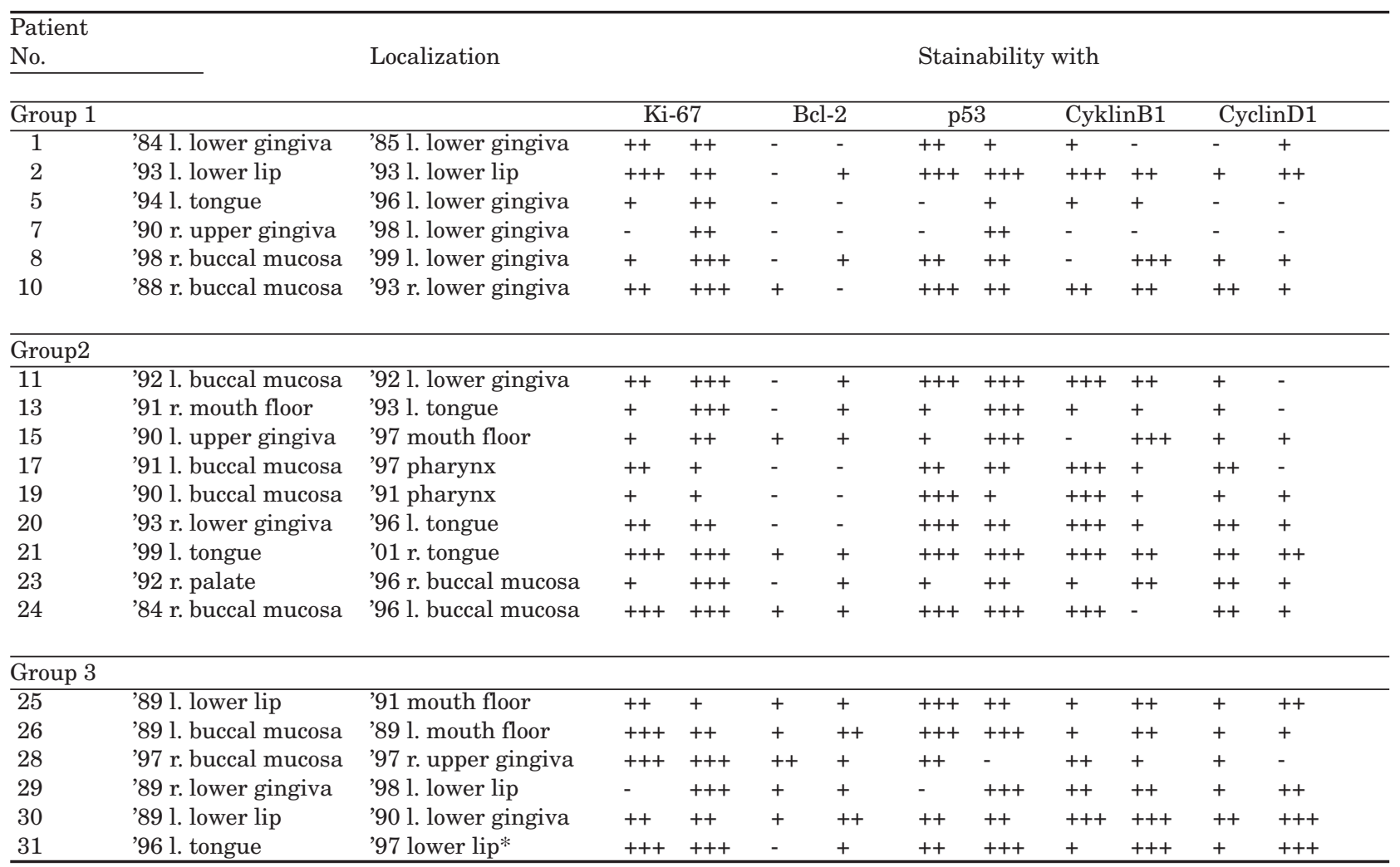

* near the vestibulum oris; l, left; r, right; +++, strong staining; ++, moderate staining; +, slight staining; -, no reaction 
Fifty-two of 69 tumors (75.4\%) showed different stainability between Cyclin B1 and Cyclin D1. Degree of expression of Cyclin B1 and Cyclin D1 was different between metachronously or synchronously multiple OSCCs. These results indirectly indicate the possibility of polyclonal development of the tumors in multiple OSCCs. The present study also suggests that multiple OSCCs in the distinct separate portions are not very high in incidence nor in carcinogens which affect independently the squamous epithelium at multiple sites, leading to numerous and independent growths of multifocal non-related tumors.

\section{References}

1. Slaughter DP, Southwick HW and Smejkal W. "Field cancerization" in oral stratified squamous epithelium. Cancer 1953; 6: $963-8$.

2. Braakhuis B.J.M., Tabor MP, Leemans CR, et al. Second primary tumors and field cancerization in oral and oropharyngeal cancer: Molecular techniques provide new insights and definitions. Head \& Neck 2002; 24: 198-206.

3. Worsham MJ, Wolman SR, Carey TE, et al. Common clonal origin of synchronous primary head and neck squamous cell carcinomas: analysis by tumor karyotypes and fluorescence in situ hybridization. Human Pathology 1995; 26: 251-61.

4. Bedi GC, Westra WH, Gabrielson E, et al. Multiple head and neck tumors: evidence for a common clonal origin. Cancer Res 1996; 56: 2484-7.

5. Califano J, van der Riet P, Westra W, et al. Genetic progression model for head and neck cancer: implication for field cancerization. Cancer Res 1996; 56: 2488-92.

6. Garcia SB, Park HS, Novelli M, et al. Field cancerization, clonality and epithelial stem cells: the spread of mutated clones in epithelial sheets. J Pathol 1999; 187: 61-81.

7. van Oijen MG, vd Straat FG, Tilanus MGJ, et al. The origins of multiple squamous cell carcinomas in the aerodigestive tract. Cancer 2000; 88: 884-93.

8. Tabor MP, Brakenhoff RH, van Houten VMM, et al. Persistence of genetically altered field in head and neck cancer patients: biological and clinical implications. Clin Cancer Res
Table 6: Different expression rate of each antibody between two distinctly separate OSCCs

\begin{tabular}{lcc}
\hline Antibody & No. of OSCCs & $\%$ \\
\hline Ki-67 & 13 & 61.9 \\
Bcl-2 & 10 & 47.6 \\
p53 & 12 & 57.1 \\
CyclinB1 & 15 & 71.4 \\
CyclinD1 & 14 & 66.7 \\
\hline
\end{tabular}

OSCCs: oral squamous cell carcinomas

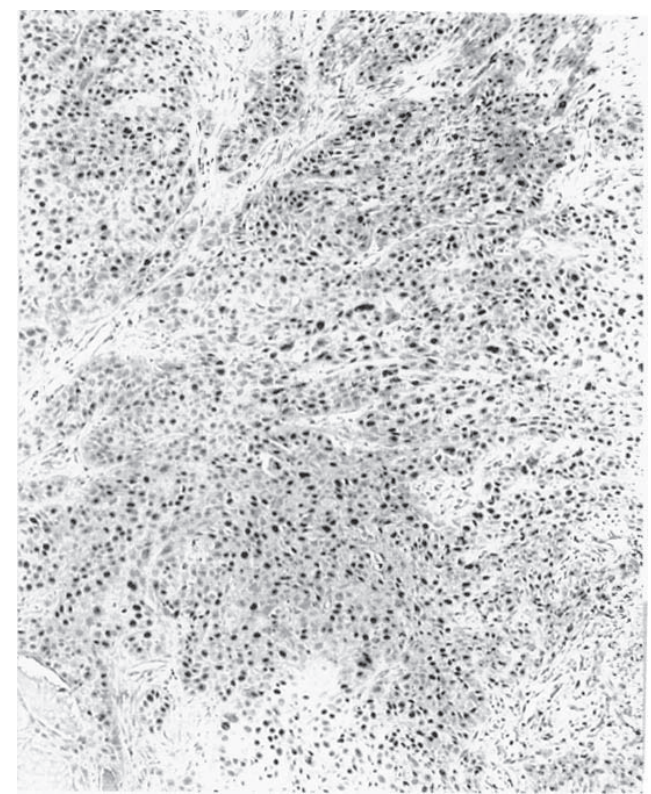

Fig. 3: Comparison of immunohistochemical stainability of Cyclin B1 of OSCCs between two distinctly separate sites in Case 24 .

Left: In SCC of right buccal mucosa in '84, positive cells are diffusely seen. ( 100)

Right: In SCC of left buccal mucosa in '96, tumor cells are not stained. ( 100) 
2001; 7: 1523-32.

9. Partridge M, Pateromichelakis S, Phillips E, et al. Profiling clonality and progression in multiple premalignant and malignant oral lesions identifies a subgroup of cases with a distinct presentation of squamous cell carcinoma. Clin Cancer Res 2001; 7: 1860-6.

10. Clifano J, Wester WH, Koch W, et al. Unknown primary head and neck squamous cell carcinoma: molecular identification of the site of origin. J Nat Cancer Inst 1999; 91: 599-604.

11. Jang SJ, Chiba I, Hirai A, et al. Multiple oral squamous epithelial lesions: are they genetically related? Oncogene 2001; 20: $2235-42$.

12. Warren S and Gates O. Multiple primary malignant tumors: a survey of the literature and statistical study. Am J Cancer 1932; 16: 1358-414.

13. Scholes AG, Woolgar JA, Boyle MA, et al. Synchronous oral carcinoma: independent or common clonal origin? Cancer Res 1998; 58: 2003-6.

14. van Oijen MGCT and Slootweg PJ. Oral field cancerization: carcinogen-induced independent events or micrometastatic deposits? Cancer Epidemiol Biomakers Prev 2000; 9: 249-56.

15. Califano J, van der Riet P, Westra W, et al. Genetic progression model for head and neck cancer: implications for field cancerization. Cancer Res 1996; 56: 2488-92.

16. Franklin WA, Gazdar AF, Haney J, et al. Widely dispersed p53 mutation in respiratory epithelium. J Clin Inves 1997; 100: $213-7$

17. Waridel F, Estreicher A, Bron L, et al. Field cancerization and polyclonal p53 mutation in the upper aerodigestive tract. Oncogene 1997; 14: 163-9.

18. Tian D, Feng Z, Hanley NM, et al. Multifocal accumulation of p53 protein in esophageal carcinoma: evidence for field cancerization. Int J Cancer 1998; 78: 568-75.

19. Kropveld A, Rozemuller EH, Leppers FGJ, et al. Sequencing analysis of RNA and DNA of Exons 1 through 11 shows p53 gene alterations to be present in almost $100 \%$ of head and neck squamous cell carcinoma. Laboratory investigation 1999; 79: 347-52.

20. Chung KY, Mukhopadhyay T, Kim J, et al. Discordant p53 gene mutation in primary head and neck cancers and corresponding second primary cancers of the upper aerodigestive tract. Cancer Res 1993; 53: 1676-83.
21. Nees M, Homann N, Discher H, et al. Expression of mutated p53 occurs in tumor-distant epithelia of head and neck cancer patients: a possible molecular basis for the development of multiple tumors. Cancer Res 1993; 53: 4189-96.

22. Khuri FR, Kim ES, Lee JJ, et al. The impact of smoking status, disease stage, and index tumor site on second primary tumor incidence and tumor recurrence in the head and neck retinoid chemoprevention trial. CEBP 2001; 10: 823-9.

23. Lydiatt WM, Anderson PE, Bazzana T, et al. Molecular support for field cancerization in the head and neck. Cancer 1998; 82: $1376-80$.

24. Roz L, Wu CL, Porter S, et al. Allelic imbalance on chromosome $3 p$ in oral dysplastic lesions: an early event in oral carcinogenesis. Cancer Res 1996; 56: 1228-31.

25. Homann N, Nees M, Conradt C, et al. Overexpression of p53 in Tumor-distant epithelia of head and neck cancer patients is associated with an increased incidence of second primary carcinoma. Clin Cancer Res 2001; 7: 290-6.

26. Raybaud-Diogene H, Tetu B, Morecy R, et al. p53 overexpression in head and neck squamous cell carcinoma: review of the literature. Oral Oncol Eur J Cancer 1996; 32B: 143-9.

27. Xie X, Clausen O, Angelis $\mathrm{P}$, et al. The prognostic value of spontaneous apotosis, Bax, Bcl-2, and p53 in oral squamous carcinoma of the tongue. Cancer 1999; 86: 913-20.

28. Toyoshima F, Moriguchi T, Wada A, et al. Nuclear export of cyclin B1 and its possible role in the DNA damage-induced $\mathrm{G}_{2}$ checkpoint. EMBOJ 1998; 17: 2728-35.

29. Hassan KA, El-Naggar AK, Soria JC, et al. Clinical significiance of cyclin $\mathrm{B} 1$ protein expression in squamous cell carcinoma of the tongue. Clin Cancer Res 2001; 7: 2458-62.

30. Kushner J, Bradley G, Young B, et al. Aberrant expression of cyclin A and cyclin B1 proteins in oral carcinoma. J Oral Pathol Med 1999; 28: 77-81.

31. Schoelch ML, Regezi JA, Dekker NP, et al. Cell cycle proteins and the development of oral squamous cell carcinoma. Oral Oncol 1999; 35: 333-42

(Accepted for publication March 13, 2003) 